Hooper, A., \& Burnett, J. (2014). Update on primary hypobetalipoproteinemia. Current Atherosclerosis Reports, 16(7)

(O)Springer Science+Business Media New York 2014

This is pre-copy-editing, author-produced version of an article accepted for publication in Current Atherosclerosis Reports following peer review.

The final publication is available at Springer via http://dx.doi.org/10.1007/s11883-014$\underline{0423-3}$

This version was made available in the UWA Research Repository on 1 July 2015 in compliance with the publisher's policies on archiving in institutional repositories.

Use of the article is subject to copyright law 


\title{
Update on primary hypobetalipoproteinemia
}

\author{
Amanda J. Hooper ${ }^{1-3}$, John R. Burnett ${ }^{1-2}$ * \\ ${ }^{1}$ Department of Clinical Biochemistry, PathWest Laboratory Medicine WA, Royal Perth \\ Hospital, Perth, Australia \\ ${ }^{2}$ School of Medicine \& Pharmacology, University of Western Australia, Perth, Australia \\ ${ }^{3}$ School of Pathology \& Laboratory Medicine, University of Western Australia, Perth, Australia \\ * Corresponding author: Dr. John R. Burnett, Department of Clinical Biochemistry, PathWest \\ Laboratory Medicine WA, Royal Perth Hospital, Wellington Street, GPO Box X2213, Perth, \\ WA 6847, Australia.
}

Phone: +61-8-9224-3121 ; Fax: +61-8-9224-1789 ; Email: john.burnett@health.wa.gov.au 
Abstract Primary hypobetalipoproteinemia refers to an eclectic group of inherited lipoprotein disorders characterized by low or absent low density lipoprotein (LDL)-cholesterol and apolipoprotein B concentrations in plasma. Abetalipoproteinemia and homozygous familial hypobetalipoproteinemia, while caused by mutations in different genes, are clinically indistinguishable. A framework for the clinical follow-up and management of these two disorders has been recently proposed, focusing on monitoring of growth in children and preventing complications by providing specialized dietary advice and fat-soluble vitamin therapeutic regimens. Other recent publications on familial combined hypolipidemia suggest that while a reduction of ANGPTL3 activity may improve insulin sensitivity, complete deficiency also reduces serum cholesterol efflux capacity and increases the risk of early vascular atherosclerotic changes, despite low LDL-cholesterol levels. Specialist laboratories offer exon-by-exon sequence analysis for the molecular diagnosis of primary hypobetalipoproteinemia. In the future, massively parallel sequencing of panels of genes involved in dyslipidemia may play a greater role in the diagnosis of these conditions.

\section{Keywords:}

Abetalipoproteinemia, apolipoprotein B, chylomicron retention disease, combined hypolipidemia, familial hypobetalipoproteinemia, hypobetalipoproteinemia, low density lipoprotein 


\section{Introduction}

The term primary hypobetalipoproteinemia (HBL) refers to an eclectic group of inherited lipoprotein disorders characterized by low ( $<5^{\text {th }}$ percentile for age and sex in the population) or absent low density lipoprotein (LDL)-cholesterol and apolipoprotein (apo) B concentrations in plasma, depending on the gene involved and mode of inheritance of the condition, together with the severity of the mutation or mutations present [1].

Abetalipoproteinemia (ABL; OMIM 200100) is a very rare ( $<1$ in one million) recessive condition characterized by the virtual absence of apoB-containing lipoproteins in plasma that typically presents early in life with the clinical manifestations of fat malabsorption, steatorrhea and failure to thrive, progressing to ophthalmological and neurological abnormalities [2]. ABL results from mutations in both alleles of the microsomal triglyceride transfer protein gene (MTTP), a molecular chaperone critical for the formation of triglyceride-rich lipoproteins, namely very low density lipoprotein (VLDL) and chylomicrons $(\mathrm{CM})$.

Familial HBL (FHBL; OMIM 107730) is rare ( 1 in 1000 to 3000) co-dominant condition characterized by low levels of LDL-cholesterol and apoB caused by a mutation in the $A P O B$ gene, usually giving rise to a truncated apoB protein [3]. Affected individuals are usually asymptomatic, but are at increased risk of fatty liver disease. Inheritance of two mutations in $A P O B$ (compound heterozygous or homozygous FHBL) is clinically indistinguishable from ABL.

Loss-of-function mutations in PCSK9 result in decreased LDL-cholesterol and apoB levels in a gene dose-dependent manner, leading to a lifetime low risk of cardiovascular disease [4]. Heterozygous PCSK9 nonsense mutations are found in 2\% of Africans and AfricanAmericans [5, 6], giving an estimated homozygosity of $\sim 1$ in 10,000. 
CM retention disease (CMRD; OMIM 246700) is a very rare ( $<1$ in one million) recessive condition characterized by the accumulation of lipid droplets within the enterocytes and the selective absence of apoB-48 containing particles from plasma. CMRD is caused by two mutations in $S A R 1 B$, the gene product of which is critical for the intracellular trafficking of CM particles [7]. Clinical manifestations of CMRD include fat malabsorption, diarrhea, abdominal distension, vomiting, and failure to thrive.

Mutations in ANGPTL3 are associated with familial combined hypolipidemia (OMIM 605019), a recessive condition characterized by a global reduction in plasma lipoproteins [8, 9].

In this report, we provide an update on primary HBL.

\section{Primary hypobetalipoproteinemia}

Lipoprotein disorders causing primary $\mathrm{HBL}$ can be classified depending on the lipid biochemical phenotype, gene involved and mode of inheritance of the condition, together with the severity of the mutation or mutations present (Table 1).

\section{Abetalipoproteinemia}

In 1992, the role of MTTP was first implicated in ABL (also known as Bassen-Kornzweig syndrome), when its activity was undetectable in intestinal biopsies of individuals with ABL [10]. Mutations in the MTTP gene on chromosome 4q22-24 were subsequently described with ABL patients carrying two defective copies [11]. MTTP encodes an 894 amino acid protein, which forms a heterodimer with the protein disulfide isomerase (PDI), thus facilitating the transfer of lipids on to nascent apoB by a shuttle mechanism [12]. Deficient MTTP activity targets the apoB for degradation, preventing the secretion of triglyceride-rich lipoproteins. Mutations in MTTP may disrupt MTTP formation, interfere with its association with PDI, or affect its ability to transfer lipids [13, 14]. 
ABL is associated with a variety of clinical manifestations affecting multiple organ systems. Fat malabsorption is a central feature of ABL and is usually observed in the neonatal period with steatorrhea, vomiting, abdominal distension and failure to thrive, and later in life, progression to atypical retinitis pigmentosa and spinocerebellar ataxia [15]. The gastrointestinal symptoms found in $\mathrm{ABL}$ usually subside with age, in part, due to the avoidance of dietary fat in these patients [16]. The duodenal mucosa appears yellow on endoscopy due to intestinal lipid accumulation [17], with histology showing normal villi with enterocytes that are distended with lipid droplets.

Acanthocytes comprise up to 50\% or more of circulating erythrocytes in ABL [16] and result from either vitamin E deficiency or an altered membrane lipid composition. Other hematological abnormalities include low erythrocyte sedimentation rates, decreased red cell survival, anemia, hyperbilirubinemia and hemolysis, and increased international normalized ratio due to vitamin $\mathrm{K}$ deficiency. Liver involvement in $\mathrm{ABL}$ includes hepatomegaly with abnormal transaminases. Liver biopsies in ABL have shown marked hepatic steatosis [15], which can progress to steatohepatitis, fibrosis and cirrhosis. The neuromuscular manifestations of ABL typically begin in the first or second decade of life, affecting both the central and peripheral nervous system. Neurological signs which relate to vitamin E deficiency include the progressive loss of deep tendon reflexes, vibratory sense and proprioception, muscle weakness and, eventually, a Friedrich’s-like form of ataxia [18]. Ophthalmological findings tend to be variable with many ABL patients asymptomatic until adulthood [16]. Loss of night vision and/or colour vision tends to occur early in the course of disease. Fundoscopic examination may reveal an atypical pigmentation of the retina, which can lead to slowly enlarging scotomas that may result in blindness.

The standard treatment for ABL is dietary fat restriction with replacement of fatsoluble vitamins, however, this fails to completely control or cure this condition $[1,15,16$, 
19]. A low-fat diet ( $<30 \%$ of total calories) will eliminate steatorrhea and allows absorption of other nutrients essential for growth and development. High dose oral vitamin E supplementation (100-300 $\mathrm{mg} / \mathrm{kg} /$ day) is recommended to halt the progression of the neurological disease, however, serum levels do not fully normalize [15, 16]. Patients need to be followed regularly for evaluation of symptoms, complications, and to monitor compliance with therapy [20].

Supplementation with a combination of high dose vitamins $\mathrm{E}$ and $\mathrm{A}$ is effective in reducing retinal degeneration [21]. Patients treated with high dose vitamin E from the age of 16 months do not develop neurological or retinal features, while progression is halted or sometimes even reversed in older patients who already show symptoms of neurological dysfunction [22]. Although serum vitamin $\mathrm{E}$ is usually undetectable in untreated ABL, supplementation results in trace concentrations, with normal levels in adipose tissue [23]. Erythrocyte and platelet vitamin E have also been used to assess tissue vitamin E status [24]. Although vitamin D and K deficiencies are inconsistent findings in ABL, oral replacement should be considered, along with other supplementary nutrients such as iron and folate if required.

A framework for the clinical follow-up and management of ABL (and compound heterozygous and homozygous FHBL) has been recently proposed, focusing on monitoring of growth in children and detecting, and preventing complications in all ABL subjects by providing specialized dietary advice and fat-soluble vitamin therapeutic regimens [20].

\section{Familial hypobetalipoproteinemia}

Mutations in the $A P O B$ gene on chromosome 2p23-24 either abolish or interfere with the translation of full-length apoB and cause FHBL [25-27]. The majority of mutations are nucleotide substitutions and deletions in exon 26. The resulting apoB truncations have traditionally been named according to a centile system. In addition, R463W and several other 
missense mutations in the $\mathrm{N}$-terminal $\beta \alpha 1$ domain of apoB have also been described [28-30]. R463W causes impaired secretion of VLDL by defective intracellular transport and enhanced binding of the mutant protein to MTTP, leading to FHBL [28].

In patients with FHBL, truncated forms of apoB are produced at lower rates than apoB-100 and have reduced lipid content [31]. As truncated apoBs shorter than apoB-30 are not detectable in plasma, this appears to be the minimum length of apoB that is required for lipoprotein assembly. Truncations shorter than this are unable to be sufficiently lipidated and are targeted for intracellular degradation [32].

FHBL can also be caused by mutations in PCSK9, which encodes a protease that binds to the LDL-receptor and targets it for lysosomal degradation within hepatocytes [33]. Nonsense and other loss-of-function PCSK9 missense mutations increase the number of LDL-receptors on the cell surface, thereby reducing both circulating LDL-cholesterol concentrations and coronary heart disease risk. Heterozygous carriers of the PCSK9 nonsense mutations Y142X and C679X had LDL-cholesterol reduced by 28\%, with a corresponding reduction in coronary heart disease risk of $88 \%$ compared to non-carriers [4]. A compound heterozygote and a homozygote for PCSK9 nonsense mutations have been described, each healthy and with a very low LDL-cholesterol at $~ 0.4 \mathrm{mmol} / \mathrm{L}[6,34]$. Human kinetic studies have confirmed that loss-of-function PCSK9 mutations increase the fractional catabolic rate of LDL [35].

Although patients with heterozygous $A P O B$-linked FHBL are often asymptomatic, many develop fatty liver [36, 37]. FHBL might represent a longevity syndrome due to a lower lifetime exposure to atherogenic apoB-containing lipoproteins. However, only the surrogate markers carotid intima-media thickness (CIMT) and distal common carotid arterial wall stiffness have been used to demonstrate the cardioprotective effects of FHBL in humans [38]. 
The long-term consequences of the increased liver transaminases and hepatic steatosis seen in heterozygous FHBL are unknown. FHBL heterozygotes have three- to five-fold greater liver fat content compared to control subjects [37, 39, 40]. Regular biochemical monitoring and liver imaging is recommended, given the potential to progress to cirrhosis, particularly in the presence of known risk factors, such as alcohol, caloric excess and liver injury [26, 41-43].

The clinical and biochemical features of homozygous (and compound heterozygous) FHBL are, in general, indistinguishable from those of ABL. Dietary fats should be restricted to prevent steatorrhea, and long-term high-dose vitamin $\mathrm{E}$ and $\mathrm{A}$ supplementation given to prevent or at least slow the progression of neuromuscular and retinal degenerative disease $[16,21]$

\section{Chylomicron retention disease}

Mutations in SAR1B, a member of the Sar1-ADP-ribosylation factor family of small GTPases that control the intracellular trafficking of proteins, are the cause of CMRD [44]. SAR1B is needed for the fusion of the intestine specific pre-CM transport vesicle to the Golgi apparatus, allowing transport of CM through the cellular secretory pathways [45]. Mutations in $S A R 1 B$ result in the inability to secrete $\mathrm{CM}$, resulting in the accumulation of lipid droplets within the enterocytes.

CMRD presents shortly after birth with diarrhea, fat malabsorption, and failure to thrive, with vomiting and abdominal distension often present [7, 46]. Acanthocytosis is rare and may be transient. Hepatomegaly and hepatic steatosis may develop in some patients, along with fat-soluble vitamin deficiencies and their manifestations. In contrast to ABL and FHBL, cirrhosis has not been reported in CMRD. The duodenal mucosa appears white on 
endoscopy with vacuolization of enterocytes in intestinal villi of normal structure present on histology, similar to the findings in ABL.

Patients with CMRD improve within a days or weeks on commencement of a low fat diet of polyunsaturated fatty acids [7]. No relationship has been found between liver transaminases, hepatomegaly and hepatic steatosis. Neurological features include hyporeflexia and loss of proprioception in adolescents through to ataxia, myopathy and sensory neuropathy in adults.

There are no specific recommendations for the follow-up or treatment of CMRD, with therapeutic regimens currently based on those recommended for ABL.

\section{Familial combined hypolipidemia}

Homozygosity (or compound heterozygosity) for ANGPTL3 gene mutations are associated with combined hypolipidemia, which is characterized by extremely low plasma levels of LDL-cholesterol, HDL-cholesterol and triglyceride [9]. The function of ANGPTL3 appears to be the reversible inhibition of lipase activity [47, 48]. The disruption of ANGPTL3 production increases lipolysis, enhancing clearance of lipoproteins and decreasing circulating lipid concentrations.

The Italian town of Campodimele has been the focus of studies into familial combined hypolipidemia. In 1991, a three-generation family was described with a dominant form of HBL that was not due to a mutation in APOB [49]. The ANGPTL3 S17X mutation was subsequently identified in the proband of this family and in an additional eight local families [50]. The prevalence of ANGPTL3 variants in the population was estimated to be $9.4 \%[50]$.

A pooled analysis of ANGPTL3 mutation carriers including 14 homozygotes, 8 compound heterozygotes and 93 heterozygotes confirmed that heterozygotes as well as 
homozygotes had a significant reduction of all plasma lipoproteins compared to 402 controls [51]. Consistent with earlier reports showing that there was no difference in the prevalence of elevated plasma concentrations of liver enzymes [50], the prevalence of fatty liver was not different among groups [51]. However, diabetes and cardiovascular disease were absent in homozygotes, raising the possibility that absence of ANGPTL3 is protective for these conditions.

Recently it was reported that ANGPTL3 S17X homozygotes showed significantly lower plasma insulin concentrations and a trend towards lower plasma glucose [52]. The absence of Angptl3 results in an increase in lipoprotein lipase activity and reduced plasma free fatty acid levels, which may improve tissue insulin action.

Surprisingly, while partial ANGPTL3 deficiency (as seen in heterozygous mutation carriers) is not associated with vascular changes, homozygosity leading to complete ANGPTL3 deficiency may be associated with an increased risk of developing early vascular atherosclerotic changes despite low plasma LDL-cholesterol levels [53]. Homozygotes showed an increased CIMT, with a $0.19 \mathrm{~mm}$ higher average CIMT and $0.54 \mathrm{~mm}$ higher maximum CIMT, and a trend towards lower brachial flow-mediated dilatation. This may relate to impaired HDL function, as homozygotes also showed a reduction in serum cholesterol efflux capacity.

The prevalence of ANGPTL3 mutations giving rise to a combined hypolipidemia phenotype in subjects with severe primary HBL is about 10\% [54]. Of subjects with total cholesterol $<2^{\text {nd }}$ percentile, those with HDL-cholesterol $<2^{\text {nd }}$ decile may be carrying ANGPTL3 mutations, whereas those with higher HDL-cholesterol may be carrying APOB mutations [54].

\section{An approach to the patient with primary hypobetalipoproteinemia}


The usual biochemical trigger for the investigation of suspected primary HBL is the finding of marked hypocholesterolemia with plasma LDL-cholesterol and apoB concentrations below the 5th percentile for age and sex [1]. A personal and family history should be taken and a physical examination conducted. Secondary causes of HBL, such as severe chronic liver disease, chronic pancreatitis, cystic fibrosis, end-stage renal disease, hyperthyroidism, cachexia and malabsorption should be excluded, and clinical manifestations, such as fat malabsorption, growth failure, fat-soluble vitamin deficiency, fatty liver disease, and neurological and ophthalmological dysfunction sought.

Patients with ABL (and compound heterozygous and homozygous FHBL) will have very low plasma total cholesterol and generally low triglyceride concentrations. LDLcholesterol, when measured by direct methods, and apoB, will be absent or very low. Vitamin E levels will also be very low, and acanthocytosis may be observed on peripheral blood smear.

Patients with heterozygous FHBL typically have plasma LDL-cholesterol and apoB concentrations that are one quarter to one third of normal. The reasons for these lower-thanexpected levels may include decreased hepatic secretion of the apoB-containing lipoproteins, or the up-regulation of the LDL-receptor, resulting in an enhanced clearance rate for VLDL and LDL particles produced by the normal allele [31].

Subjects who carry a single MTTP mutation may have normal plasma lipids or may have LDL-cholesterol and apoB concentrations similar to those seen in heterozygous FHBL.

In CMRD, total cholesterol, LDL-cholesterol and HDL-cholesterol are low, but triglyceride levels are generally normal. The low plasma LDL- and HDL-cholesterol are a consequence of low rates of apoB-100 and apoA-I production [55]. An increased plasma creatine kinase concentration of up to five times normal may be seen from infancy, along with deficiencies in fat-soluble vitamins [7]. 
If concomitant reductions in triglyceride and HDL-cholesterol concentrations are observed, this is suggestive of familial combined hypolipidemia.

\section{Molecular tests}

Sequencing of the HBL genes MTTP, APOB, SAR1B, PCSK9 and ANGPTL3 is available in specialist laboratories. These molecular assays are usually designed to target exonic regions as well as at least 20 base pairs of flanking intronic sequence in order to capture any potential splice site mutations. Where two mutations are identified, testing of the patient's parents is recommended to confirm that the mutations originate from two different chromosomes.

Western blotting can be used to detect truncated apoB species that are $>30 \%$ of fulllength protein size. DNA sequencing of the region in $A P O B$ where the mutation is estimated to occur can then be performed. However, as truncated apoB species shorter than apoB-30 are not detectable in plasma, in the situation of a negative Western blot result then sequencing of the first $30 \%$ of the $A P O B$ gene (exons 1 to 25 ) should be performed.

In patients with $\mathrm{ABL}$ where the inheritance pattern is unclear or MTTP mutations unable to be identified, then the $A P O B$ gene should also be sequenced given the clinical and biochemical similarities with compound heterozygous and homozygous FHBL. Likewise, in patients with homozygous FHBL, the MTTP gene could be sequenced in the event where $A P O B$ mutations are unable to be found. Alternatively, high-throughput sequencing technology is emerging as a means for screening multiple genes for mutations. This may prove more cost effective than traditional Sanger sequencing, particularly where multiple genes may need to be sequenced and when the $A P O B$ gene is involved, which needs over 40 primer sets to cover the whole coding region. Recently, a next-generation resequencing approach for the diagnosis of monogenic dyslipidemias has been described; this targets a customized panel of 73 genes, including those associated with HBL, and has the potential to 
diagnose patients rapidly and at much lower cost than traditional Sanger or whole exome sequencing [56].

\section{Conclusion}

In this report, we provide an update on primary HBL, an eclectic group of inherited lipoprotein disorders characterized by low or absent LDL-cholesterol and apoB concentrations in plasma, depending on the gene involved and mode of inheritance of the condition, together with the severity of the mutation or mutations present. Some of these lipoprotein disorders such as ABL (and compound heterozygous and homozygous FHBL) are associated with clinical manifestations of fat malabsorption, growth failure, fat-soluble vitamin deficiency, fatty liver disease, and neuro-ophthalmological dysfunction, whereas others such as heterozygous FHBL may be asymptomatic. Molecular testing of MTTP, APOB, SAR1B, PCSK9 and ANGPTL3 genes is available in specialist laboratories. A framework for the clinical follow-up and management of ABL (and compound heterozygous and homozygous FHBL) has been recently proposed, focusing on monitoring of growth in children and detecting, and preventing complications in all ABL subjects by providing specialized dietary advice and fatsoluble vitamin therapeutic regimens.

\section{Acknowledgments}

This work was supported by National Health and Medical Research Council Project Grant 1010133 (to AJH and JRB) and a Practitioner Fellowship from the Royal Perth Hospital Medical Research Foundation (to JRB).

\section{References}

[1] Hooper AJ, van Bockxmeer FM, Burnett JR. Monogenic hypocholesterolaemic lipid disorders and apolipoprotein B metabolism. Crit Rev Clin Lab Sci 2005; 42:515-545. 
[2] Burnett JR, Bell DA, Hooper AJ, Hegele RA. Clinical utility gene card for: Abetalipoproteinaemia. Eur J Hum Genet 2012; 20:doi: 10.1038/ejhg.2012.1030.

[3] Burnett JR, Bell DA, Hooper AJ, Hegele RA. Clinical utility gene card for: Familial Hypobetalipoproteinaemia (APOB). Eur J Hum Genet 2012; 20:doi: 10.1038/ejhg.2012.1085.

[4] Cohen JC, Boerwinkle E, Mosley TH, Jr., Hobbs HH. Sequence variations in PCSK9, low LDL, and protection against coronary heart disease. N Engl J Med 2006; 354:12641272.

[5] Cohen J, Pertsemlidis A, Kotowski IK, Graham R, Garcia CK, Hobbs HH. Low LDL cholesterol in individuals of African descent resulting from frequent nonsense mutations in PCSK9. Nat Genet 2005; 37:161-165.

[6] Hooper AJ, Marais AD, Tanyanyiwa DM, Burnett JR. The C679X mutation in PCSK9 is present and lowers blood cholesterol in a Southern African population. Atherosclerosis 2007; 193:445-448.

[7] Peretti N, Sassolas A, Roy CC, et al. Guidelines for the diagnosis and management of chylomicron retention disease based on a review of the literature and the experience of two centers. Orphanet J Rare Dis 2010; 5:24.

[8] Hooper AJ, Burnett JR. Recent developments in the genetics of LDL deficiency. Curr Opin Lipidol 2013; 24:111-115.

[9] Musunuru K, Pirruccello JP, Do R, et al. Exome sequencing, ANGPTL3 mutations, and familial combined hypolipidemia. N Engl J Med 2010; 363:2220-2227.

[10] Wetterau JR, Aggerbeck LP, Bouma ME, et al. Absence of microsomal triglyceride transfer protein in individuals with abetalipoproteinemia. Science 1992; 258:999-1001.

[11] Shoulders CC, Brett DJ, Bayliss JD, et al. Abetalipoproteinemia is caused by defects of the gene encoding the $97 \mathrm{kDa}$ subunit of a microsomal triglyceride transfer protein. Hum Mol Genet 1993; 2:2109-2116.

[12] Hussain MM, Rava P, Walsh M, Rana M, Iqbal J. Multiple functions of microsomal triglyceride transfer protein. Nutr Metab (Lond) 2012; 9:14.

[13] Ohashi K, Ishibashi S, Osuga J, et al. Novel mutations in the microsomal triglyceride transfer protein gene causing abetalipoproteinemia. J Lipid Res 2000; 41:1199-1204.

[14] Rehberg EF, Samson-Bouma ME, Kienzle B, et al. A novel abetalipoproteinemia genotype. Identification of a missense mutation in the $97-\mathrm{kDa}$ subunit of the microsomal triglyceride transfer protein that prevents complex formation with protein disulfide isomerase. J Biol Chem 1996; 271:29945-29952.

[15] Berriot-Varoqueaux N, Aggerbeck LP, Samson-Bouma M, Wetterau JR. The role of the microsomal triglyceride transfer protein in abetalipoproteinemia. Annu Rev Nutr 2000; 20:663-697.

[16] Kane JP, Havel RJ, Disorders of the biogenesis and secretion of lipoproteins containing the B apolipoproteins. In: Scriver CR, Beaudet AL, Sly WS, et al., editors. The Metabolic and Molecular Bases of Inherited Disease. New York: McGraw-Hill, 2001: 2717-2752.

[17] Delpre G, Kadish U, Glantz I, Avidor I. Endoscopic assessment in abetalipoproteinemia (Bassen-Kornzweig-syndrome). Endoscopy 1978; 10:59-62.

[18] Tanyel MC, Mancano LD. Neurologic findings in vitamin E deficiency. Am Fam Physician 1997; 55:197-201.

[19] Zamel R, Khan R, Pollex RL, Hegele RA. Abetalipoproteinemia: two case reports and literature review. Orphanet J Rare Dis 2008; 3:19.

[20] Lee J, Hegele RA. Abetalipoproteinemia and homozygous hypobetalipoproteinemia: a framework for diagnosis and management. J Inherit Metab Dis 2014 (in press). 
- Discusses the diagnosis, assessment, treatment and follow-up of abetalipoproteinemia and homozygous hypobetalipoproteinemia.

[21] Chowers I, Banin E, Merin S, Cooper M, Granot E. Long-term assessment of combined vitamin $A$ and $E$ treatment for the prevention of retinal degeneration in abetalipoproteinaemia and hypobetalipoproteinaemia patients. Eye 2001; 15:525-530.

[22] Muller DP. Vitamin E and neurological function. Mol Nutr Food Res 2010; 54:710718.

[23] Kayden HJ, Hatam LJ, Traber MG. The measurement of nanograms of tocopherol from needle aspiration biopsies of adipose tissue: normal and abetalipoproteinemic subjects. J Lipid Res 1983; 24:652-656.

[24] Clarke MW, Hooper AJ, Headlam HA, Wu JH, Croft KD, Burnett JR. Assessment of tocopherol metabolism and oxidative stress in familial hypobetalipoproteinemia. Clin Chem 2006; 52:1339-1345.

[25] Schonfeld G, Lin X, Yue P. Familial hypobetalipoproteinemia: genetics and metabolism. Cell Mol Life Sci 2005; 62:1372-1378.

[26] Tarugi P, Averna M. Hypobetalipoproteinemia: genetics, biochemistry, and clinical spectrum. Adv Clin Chem 2011; 54:81-107.

[27] Whitfield AJ, Barrett PHR, van Bockxmeer FM, Burnett JR. Lipid disorders and mutations in the APOB gene. Clin Chem 2004; 50:1725-1732.

[28] Burnett JR, Shan J, Miskie BA, et al. A novel nontruncating APOB gene mutation, R463W, causes familial hypobetalipoproteinemia. J Biol Chem 2003; 278:1344213452.

[29] Burnett JR, Zhong S, Jiang ZG, et al. Missense mutations in $A P O B$ within the betaalpha1 domain of human APOB-100 result in impaired secretion of apoB and apoB-containing lipoproteins in familial hypobetalipoproteinemia. J Biol Chem 2007; 282:24270-24283.

[30] Zhong S, Magnolo AL, Sundaram M, et al. Nonsynonymous mutations within APOB in human familial hypobetalipoproteinemia - evidence for feedback inhibition of lipogenesis and post-endoplasmic reticulum degradation of apolipoprotein B. J Biol Chem 2010; 285:6453-6464.

[31] Elias N, Patterson BW, Schonfeld G. Decreased production rates of VLDL triglycerides and apoB-100 in subjects heterozygous for familial hypobetalipoproteinemia. Arterioscler Thromb Vasc Biol 1999; 19:2714-2721.

[32] Yao ZM, Blackhart BD, Linton MF, Taylor SM, Young SG, McCarthy BJ. Expression of carboxyl-terminally truncated forms of human apolipoprotein B in rat hepatoma cells. Evidence that the length of apolipoprotein B has a major effect on the buoyant density of the secreted lipoproteins. J Biol Chem 1991; 266:3300-3308.

[33] Lambert G, Sjouke B, Choque B, Kastelein JJ, Hovingh GK. The PCSK9 decade. J Lipid Res 2012; 53:2515-2524.

[34] Zhao Z, Tuakli-Wosornu Y, Lagace TA, et al. Molecular characterization of loss-offunction mutations in PCSK9 and identification of a compound heterozygote. Am J Hum Genet 2006; 79:514-523.

[35] Cariou B, Ouguerram K, Zair Y, et al. PCSK9 dominant negative mutant results in increased LDL catabolic rate and familial hypobetalipoproteinemia. Arterioscler Thromb Vasc Biol 2009; 29:2191-2197.

[36] Ogata H, Akagi K, Baba M, et al. Fatty liver in a case with heterozygous familial hypobetalipoproteinemia. Am J Gastroenterol 1997; 92:339-342.

[37] Schonfeld G, Patterson BW, Yablonskiy DA, et al. Fatty liver in familial hypobetalipoproteinemia: triglyceride assembly into VLDL particles is affected by the extent of hepatic steatosis. J Lipid Res 2003; 44:470-478. 
[38] Sankatsing RR, Fouchier SW, de Haan S, et al. Hepatic and cardiovascular consequences of familial hypobetalipoproteinemia. Arterioscler Thromb Vasc Biol 2005; 25:1979-1984.

[39] Tanoli T, Yue P, Yablonskiy D, Schonfeld G. Fatty liver in familial hypobetalipoproteinemia: roles of the APOB defects, intra-abdominal adipose tissue, and insulin sensitivity. J Lipid Res 2004; 45:941-947.

[40] Visser ME, Lammers NM, Nederveen AJ, et al. Hepatic steatosis does not cause insulin resistance in people with familial hypobetalipoproteinaemia. Diabetologia 2011; 54:2113-2121.

[41] Hooper AJ, Adams LA, Burnett JR. Genetic determinants of hepatic steatosis in man. J Lipid Res 2011; 52:593-617.

[42] Lonardo A, Tarugi P, Ballarini G, Bagni A. Familial heterozygous hypobetalipoproteinemia, extrahepatic primary malignancy, and hepatocellular carcinoma. Dig Dis Sci 1998; 43:2489-2492.

[43] Tarugi P, Lonardo A, Ballarini G, et al. A study of fatty liver disease and plasma lipoproteins in a kindred with familial hypobetalipoproteinemia due to a novel truncated form of apolipoprotein B (APO B-54.5). J Hepatol 2000; 33:361-370.

[44] Jones B, Jones EL, Bonney SA, et al. Mutations in a Sar1 GTPase of COPII vesicles are associated with lipid absorption disorders. Nat Genet 2003; 34:29-31.

[45] Siddiqi SA, Gorelick FS, Mahan JT, Mansbach CM, 2nd. COPII proteins are required for Golgi fusion but not for endoplasmic reticulum budding of the pre-chylomicron transport vesicle. J Cell Sci 2003; 116:415-427.

[46] Peretti N, Roy CC, Sassolas A, et al. Chylomicron retention disease: a long term study of two cohorts. Mol Genet Metab 2009; 97:136-142.

[47] Nakajima K, Kobayashi J, Mabuchi H, et al. Association of angiopoietin-like protein 3 with hepatic triglyceride lipase and lipoprotein lipase activities in human plasma. Ann Clin Biochem 2010; 47:423-431.

[48] Shan L, Yu XC, Liu Z, et al. The angiopoietin-like proteins ANGPTL3 and ANGPTL4 inhibit lipoprotein lipase activity through distinct mechanisms. J Biol Chem 2009; 284:1419-1424.

[49] Fazio S, Sidoli A, Vivenzio A, et al. A form of familial hypobetalipoproteinaemia not due to a mutation in the apolipoprotein B gene. J Intern Med 1991; 229:41-47.

[50] Minicocci I, Montali A, Robciuc MR, et al. Mutations in the ANGPTL3 gene and familial combined hypolipidemia: a clinical and biochemical characterization. J Clin Endocrinol Metab 2012; 97:E1266-1275.

[51] Minicocci I, Santini S, Cantisani V, et al. Clinical characteristics and plasma lipids in subjects with familial combined hypolipidemia: a pooled analysis. J Lipid Res 2013; 54:3481-3490.

[52] Robciuc MR, Maranghi M, Lahikainen A, et al. Angptl3 deficiency is associated with increased insulin sensitivity, lipoprotein lipase activity, and decreased serum free fatty acids. Arterioscler Thromb Vasc Biol 2013; 33:1706-1713.

-Shows that although partial deficiency of ANGPTL3 did not affect lipase activity, complete deficiency decreased free fatty acids and improves insulin sensitivity.

[53] Minicocci I, Cantisani V, Poggiogalle E, et al. Functional and morphological vascular changes in subjects with familial combined hypolipidemia: an exploratory analysis. Int J Cardiol 2013; 168:4375-4378.

$\bullet \bullet$ Despite a $\sim 50 \%$ reduction in LDL-cholesterol, ANGPTL3 homozygotes had increased carotid intima-media thickness. 
[54] Noto D, Cefalu AB, Valenti V, et al. Prevalence of ANGPTL3 and APOB gene mutations in subjects with combined hypolipidemia. Arterioscler Thromb Vasc Biol 2012; 32:805-809.

[55] Ouguerram K, Zair Y, Kasbi-Chadli F, et al. Low rate of production of apolipoproteins B100 and AI in 2 patients with Anderson disease (chylomicron retention disease). Arterioscler Thromb Vasc Biol 2012; 32:1520-1525.

[56] Johansen CT, Dube JB, Loyzer MN, et al. LipidSeq: a next-generation clinical resequencing panel for monogenic dyslipidemias. J Lipid Res 2014.

- Describes the design and performance of a targeted resequencing panel which has potential to aid in molecular diagnosis of dyslipidemias including primary hypobetalipoproteinemia 
Table 1. Lipoprotein disorders causing primary hypobetalipoproteinemia.

\begin{tabular}{|l|l|l|l|l|}
\hline Lipoprotein disorder & Gene & Inheritance & Biochemical phenotype & Clinical phenotype \\
\hline Abetalipoproteinemia & MTTP & Recessive & $\begin{array}{l}\text { Absence of LDL and } \\
\text { chylomicrons, low } \\
\text { triglycerides, very low } \\
\text { vitamin E }\end{array}$ & $\begin{array}{l}\text { Variable; includes } \\
\text { failure to thrive, } \\
\text { steatorrhea, } \\
\text { progressive } \\
\text { neurological and } \\
\text { ophthalmological } \\
\text { abnormalities }\end{array}$ \\
\hline $\begin{array}{l}\text { Familial } \\
\text { hypobetalipoproteinemia }\end{array}$ & APOB & Co-dominant & $\begin{array}{l}\text { Heterozygous: LDL- } \\
\text { cholesterol <30\% levels } \\
\text { of normal for age and } \\
\text { sex } \\
\text { Homozygous: absence } \\
\text { or very low levels of } \\
\text { LDL-cholesterol, low } \\
\text { triglycerides, very low } \\
\text { vitamin E }\end{array}$ & $\begin{array}{l}\text { Heterozygous: } \\
\text { generally } \\
\text { asymptomatic, may } \\
\text { include fatty liver } \\
\text { Homozygous: } \\
\text { indistinguishable from } \\
\text { abetalipoproteinemia }\end{array}$ \\
\hline $\begin{array}{l}\text { Familial } \\
\text { hypobetalipoproteinemia }\end{array}$ & PCSK9 & Co-dominant & $\begin{array}{l}\text { Heterozygous: } ~ 40 \% \\
\text { reduction in LDL } \\
\text { Homozygous: very low } \\
\text { LDL-cholesterol }\end{array}$ & $\begin{array}{l}\text { None } \\
\text { Absence of } \\
\text { chylomicrons, } \\
\text { LDL-cholesterol <50\% } \\
\text { levels of normal for age } \\
\text { and sex }\end{array}$ \\
\hline $\begin{array}{l}\text { Chylomicron retention } \\
\text { disease }\end{array}$ & SAR1B & Recessive & $\begin{array}{l}\text { Variable; includes } \\
\text { failure to thrive, } \\
\text { steatorrhea and } \\
\text { progressive } \\
\text { neurological } \\
\text { abnormalities }\end{array}$ \\
\hline & ANGPTL3 & Recessive & $\begin{array}{l}\text { Reduced levels of all } \\
\text { plasma lipoproteins }\end{array}$ & None \\
\hline
\end{tabular}

